\title{
Die Predigt als Kommunikationsmedium
}

\author{
von Heribert Arens, Franz Richardt, Josef Schulte \\ (Homiletische Arbeitsgruppe)
}

\section{Die Predigt als Krisenphänomen kirchlicher Kommunikation}

Befrachtet mit dem Ballast einer langen Geschichte, belastet mit dem Image, einer vor-demokratischen Gesellschaftsform anzugehören, bedroht vom Schicksal der Ineffektivität, belächelt als die institutionalisierte Belanglosigkeit, eingeengt vom Auftrag, Schrift auszulegen, beschimpft als monologisch und autoritär, dennoch insgeheim gefürchtet als Medium der Indoktrination und Manipulation - von Schlagwörtern fast erschlagen, hält sich in den Kirchen ein Kommunikationsmedium mit der Hartnäckigkeit einer alten Erbtante, die sterben soll, aber nicht will: die Predigt.

In der Tat birgt die Predigt als Kommunikationsmedium manche Schwierigkeiten obwohl wöchentlich für die Sonntagspredigt viel investiert wird, sei es Zeit zur Vorbereitung, sei es Geld zur Anschaffung von Predigthilfen.

Welcher Art sind diese Schwierigkeiten? Kann man ihnen entgegenwirken - und wie? Diese Fragen sollen im folgenden beantwortet werden.

\section{Das Informationsproblem}

„Die Kirchen haben der Gesellschaft nichts Nennenswertes mehr zu sagen." So ungefähr hat sich Rudolf Augstein vor einigen Jahren geäußert. Als Analyse der Möglichkeiten ist dieser Satz abzulehnen, als Analyse des Möglichen sagt er eine Wahrheit, die den Predigern Anlaß nachzudenken sein sollte. Bestimmt man den Grad der Information mit Siegfried Maser ${ }^{1}$ nach dem Grad der Unwahrscheinlichkeit oder Unvoraussagbarkeit, den Grad der Redundanz, des Bekannten, nach dem Grad der Wahrscheinlichkeit und Voraussagbarkeit - und eine gute Mischung von Information und Redundanz sollte die Predigt sein -, dann nähern sich viele Predigten in der Tat dem Informationswert "Null“, vergleichbar den Schlagertexten oder den Reden deutscher Politiker an den letzten Tagen vor dem 19. November 1972: Ihr Inhalt ist redundant, $d$. h. überflüssig, weil bekannt.

Voraussagbar ist bei vielen Predigern bereits der Tonfall: Der Hörer erlebt ihn wie der Bundesbahnfahrgast die Drähte neben den Gleisen. Aufgehängt an den Telegrafenmasten dynamisch-melodischer Höhenakzente schwingt die Sprechmelodie von Höhepunkt zu Höhepunkt, nicht selten gemischt mit einer engagiert-intensiv-salbungsvollen Klangfarbe. "Das klingt nach Predigt", so daß auch für diesen Punkt zu Recht die Forderung Wilhelm Gössmanns besteht: Predigen, als predigte man nicht!?

Die Homiletische Arbeitsgruppe arbeitet in den Bereichen Homiletik und Sprechkunde, für die sie Lehraufträge an der Ordenshochschule der Franziskaner und Kapuziner in Münster und am Priesterseminar Osnabrüdk hat. Ein weiterer Schwerpunkt ihrer Arbeit liegt in einer Kurstätigkeit, die homiletische Themen sowie die Bereiche Gesprächsführung und Rhetorik zum Inhalt hat. Zielgruppen dieser Tätigkeit sind Prediger, Seminaristen, Pastoralassistenten, Studenten, Primaner, Pfarrgemeinderäte, Volkshochschulgruppen und Ordensleute. Die drei Mitglieder der Gruppe arbeiten an verschiedenen Predigtzeitschriften mit. Heribert Arens ist der Verfasser des Buches „Die Predigt als Lernprozeß“ (München 1972). 
Voraussagbar ist das Vokabular, besonders im Bereich der Wortarten, die die sprachlichen Muster der Sätze bilden: Sollten nicht auch wir? - So wollen denn auch wir! - Immer wieder - Stets von neuem. ... Stereotype Predigtformulierungen, in die „Bütt“ verfremdet, haben schon manchem Karnevalisten Lacherfolge verschafft.

Eng mit dem Vokabular ist der Inhalt verbunden - und voraussagbar. Der Grund: In vielen Fällen werden biblische Erzählungen nacherzählt und kommentiert. Anschließend werden dann meist in der Form von Fragen und Imperativen Applikationspfeile aus dem "heiligen Land" in die Ortsgemeinde abgeschossen. Der Leser möge sich einmal besinnen, welche Worte ihm einfallen, wenn er an das Gleichnis vom barmherzigen Samariter denkt. Es wird sich ein Vokabular einstellen, mit dem er zumindest 50\% der Predigten, die zu dieser Perikope gehalten wurden, rekonstruieren kann. Ein zweiter Grund: Manche Predigten halten sich mit Vergnügen sprachlich und inhaltlich im Denkhorizont heutigen sozialen Engagements christlicher bis marxistischer Prägung auf. Solidarität, Randsiedler, Dirnen, protestieren, Ausgestoßene, herrschende Klasse, unterdrücken u. ä. vokabelt es dann.

Die Diagnose "belanglos“ ist nicht falsch am Platz. Daraus jedoch auf mangelnde Stoßkraft der Botschaft zu schließen, wäre kurzsichtig. Es geht um ein hermeneutisches Problem. Die Hermeneutik dialektischer Theologie - theologisch zwar überholt, praktisch aber noch Sonntag für Sonntag in ökumenischer Einträchtigkeit in den Kirchen frisch und munter gepredigt - mit ihrer Lehre von der Selbstwirksamkeit des Wortes Gottes legitimiert das Nebeneinander von erlittener Situation und unverstandener, nur wiederholter Botschaft, statt zu Ubersetzung und Konkretion anzuregen. ${ }^{3}$ Damit verbunden ist die Unfähigkeit der Kirche, konkret zu werden. ${ }^{4}$ Aber erst im Konkreten ist die Ubbersetzung am Ziel, nicht im Allgemeinen. Die Prediger ziehen zwar große und typische Linien, aber gerade die sind voraussagbar. Je konkreter eine Predigt ist, desto weniger ist sie im gleichen Wortlaut wiederholbar.

Hier stehen wir vor einem Problemfeld des Kommunikationsmediums „Predigt": Die Belanglosigkeit der vermittelten bzw. postulierten Information. Sie ist bestimmt durch den Gegenstand der Kommunikation sowie durch die Beziehung des Kommunikators zu diesem Gegenstand. Um das Medium „Predigt" für diesen Bereich $\mathrm{zu}$ verbessern, ist hermeneutische und didaktische Aus- und Weiterbildung der Prediger oberstes Gebot der Stunde.

\section{Das Öffentlichkeitsproblem}

Ein zweites Problem ist die Offentlichkeit der Predigt. Offentlichkeit ist hier gemeint im Sinne der dritten Definition Dovifats ${ }^{5}$ : Nach dieser Definition kann man „Offentlichkeit" im Plural verwenden. Es gibt verschiedene Offentlichkeiten, die durch je besondere Qualifikationen gekennzeichnet sind. Nun kommt die Predigtöffentlichkeit bei der Beschreibung ihrer Qualifikationen nicht gerade gut weg. Viele sehen in ihr eine Offentlichkeit, die sich aus Menschen zusammensetzt, die Bestätigung suchen, sich nicht verunsichern lassen wollen, sich systemimmanent mit der Kommunilkationsstruktur "Einbahnkommunikation" zufriedengeben und entsprechend gesellschaftlich den vor-demokratischen Status stabilisieren. Sie wird als eine Offentlichkeit gesehen, die sich mit kirchlichen Antworten in jener Selbstverständlichkeit identifiziert, in der sie es mit menschlichen und gesellschaftlichen Fragen tun sollte. ${ }^{6}$ 
Stimmt diese Umschreibung der Predigtöffentlichkeit, dann ist Predigen ein belangloses und überflüssiges Geplauder oder eine Sisyphusarbeit, die ihren einzigen Sinn darin findet, wenig sinn-voll zu sein.

Es gibt den stumpf sinnierenden Hörer, es gibt den Hörer, der „auf den Ohren sitzt", es gibt den Wunsch nach Stabilität, den Wunsch, nichts zu verändern. Vielleicht sind die Hörer aber auch so, weil die Voraussagbarkeit vieler Predigten sie nichts anderes erwarten läßt. Ursache und Wirkung sind hier kaum zu trennen. Wenn etwa Predigtarbeit über lange Strecken gesellschaftlich nicht relevant war, Belangloses sagte, vielleicht hat sie sich ein belangloses Publikum geschaffen.

Es bleibt jedenfalls festzustellen:

- Trotz einer Anzahl volkskirchlich denkender Hörer, die der Gewohnheit näher stehen als der Reflexion, kommen viele Hörer zu den Predigten, die denkbereit, fragefreudig und auf Gedankenimpulse für ihr persönliches Leben bedacht sind.

- Die Untersuchungen Osmund Schreuders, die die Diskussion über die belanglose Hörerschaft und die damit verbundene Ineffektivität der Predigt intensiviert haben, stammen aus einer Zeit, in der die Homiletik Erkenntnisse wie die der Kommunikationsforschung oder Lernpsychologie noch nicht oder doch nur in spärlichen Ansätzen übernommen hatte. ${ }^{7}$ Es ist offen, ob diese Erkenntnisse nicht helfen können, im Rahmen von Einbahnkommunikation auch bei volkskirchlichen Hörern Lernprozesse auszulösen.

- Der Prediger schafft sich seine Hörerschaft. Wer belanglos predigt, darf auch mit einer belanglosen Offentlichkeit rechnen. Aber uns sind nicht wenige Kirchen bekannt, in denen sich eine ernst zu nehmende und kreative Offentlichkeit sammelt, weil der Prediger kreativ und anregend ist.

\section{Das Gattungsproblem}

Mit dem Stichwort „Lernprozeß“ ist ein drittes Problemfeld angesprochen: die Kommunikationsform. Ist die monologische Kommunikationsform "Rede", die sich in Jahrhunderten ihre Existenzberechtigung ersessen hat, die geeignete Form, Kommunikation zwischen Menschen einer demokratischen Gesellschaft zu ermöglichen, denen doch der Dialog zueigen ist? Ist es die geeignete Form, Informationen zu vermitteln? Es ist nachgewiesen, daß der Dialog als Form zu diesem Ziel effektiver ist. ${ }^{8}$

Aus verschiedenen Gründen vertreten darum die Verfechter der dialogischen Kommunikationsform für die Predigt ein wichtiges Anliegen. Verkündigung richtet sich an Hörer, möchte Inhalte vermitteln, möchte Botschaft in das konkrete Leben der Hörer hineinsprechen. Gerade die Tatsache, daß der Hörer in der Predigt „vorkommen soll“, wirft die Frage auf: Warum soll er dann nicht selbst reden? Warum soll er etwa Fragen, die er hat, nicht selbst stellen? Diese Fragestellung hängt mit der hermeneutischen Frage zusammen. Eine Hermeneutik, die den Hörer konsequent in den Blick nimmt, muß sich auch die Frage stellen: Soll der Hörer selbst reden bei der Predigt?o

Diese drei Schlaglichter zeigen, daß die Predigt in unserer Zeit ein Krisenphänomen ist. Will sich die Predigt nicht mit einer "Händchen-Halten-Funktion" zufriedengeben - und das darf sie nicht - , dann muß sie sich unter dem Auftrag verstehen, bei den Hörern bestimmte Ziele zu erreichen. Als Globalziel der Verkündigung, wie es sich aus einer zeit- und bibelnahen Theologie ergibt, darf man Daseinserbellung 
nennen. ${ }^{10}$ Die Botschaft des Damals soll für die heutige Situation als erhellend erfahrbar gemacht werden. Prozesse der Daseinserhellung sind aber immer auch Lernprozesse, sei es, daß ich lerne, Dinge ganz anders zu sehen, sei es, daß ich lerne, Dinge tiefer, heller, pointierter zu sehen. ${ }^{11}$

Wir wollen darum im folgenden der Frage nachgehen: Wie kann das Kommunikationsmedium "Predigt" unter den Aspekten der Lernpsychologie und der Kommunikationsform verbessert werden?

\section{Lernpsychologische Anregungen zur Sicherung der Kommunikation und zur Steigerung der Information}

Das Kommunikationsmedium „Predigt" wird ineffektiv, erstarrt zur rituellen Interaktion, wenn nur ein Kommunikationspartner aktiv ist, während der andere abgelenkt seinen Gedanken nachgeht. Kommunikation ist abgebrochen, genau wie ein Dialog nicht stattfindet, wenn der Partner $z$ war da, aber abwesend ist. Effektive Kommunikation verlangt, daß alle Partner an der Kommunikation beteiligt sind. Dabei gilt Zuhören, das auf den Gegenstand der Kommunikation gerichtet ist, als aktive Beteiligung. ${ }^{12}$

\section{Darstellung des Lernprozesses}

Interesse artikuliert sich in Fragen. Will der Kommunikator den Rezipienten interessieren, will er ihn durch Interesse am Prozeß der Einbahnkommunikation beteiligen, dann wird er nicht anders können und dürfen, als Fragen des Rezipienten aufzugreifen. Diese Forderung wird verstärkt aus der Lerntheorie: Lernprozesse werden nur ausgelöst, wenn Motivationen beim Lernenden vorhanden sind, Bedürfnisspannungen, Fragen, Wünsche, Interessen, Neigungen, die den Lernenden auf das Lernziel hin öffnen. ${ }^{13}$

Die Lernstufen, wie sie die Lernpsychologie erarbeitet hat, sind darum geeignet als Stufen, die dem Kommunikationsmedium "Predigt" hilfreich sein können, sind es doch didaktische Stufen, die bei Fragen der Hörer ansetzen und in dauernder Rückversicherung seines Interesses bis zur Informationsverarbeitung führen.

Im allgemeinen differenziert man für den reflektiert durchgeführten Lernprozeß oder für den vom Lehrer angelegten und begleiteten Lernprozeß folgende 5 Stufen $^{14}$ :

a) Motivation: Eine Frage, Bedürfnisspannung, ein Wunsch, eine Erfahrung, die Fragen birgt, wird aufgegriffen und dargestellt.

b) Problemabgrenzung: Gleichsam als abstrakte Wahrheit wird das Problem von (a) in Worte gekleidet, dem Verstand zugänglich gemacht.

c) Versuch und Irrtum: Verschiedene Lösungsversuche auf das Problem werden durchgespielt in ihren Konsequenzen.

d) Lösungsangebot: Der Lehrende macht sein Angebot, bzw. der Lernende findet die richtige Lösung.

e) Lösungsverstärkung: Die Richtigkeit dieser Lösung wird durch Erfahrungen, durch Erfolg, durch Beispiele oder Worte bestätigt. 
Ein Beispiel mag diese Stufen verdeutlichen: a) Ein Prediger erlebt, daß Hörer kaum den Inhalt seiner Predigt wiedergeben können; sie behalten nur einige schöne Formulierungen und Bilder, nicht sein Anliegen; b) Daraus entsteht für ihn das Problem: Das muß ich ändern; ich muß Wege finden, diese Ergebnisse zu verbessern; c) Er paßt sich dem Jargon der Jugend an - und merkt, daß das nichts nutzt; er predigt kürzer - aber auch das fruchtet nicht; d) Durch Zufall bespricht er mit einem Lehrer, der sich in der Lernpsychologie auskennt, sein Problem. Der erklärt ihm, wie er seine Unterrichtsstunden anlegt, etwa nach den genannten fünf Stufen. Das ist ein Lösungsangebot, von dem sich der Prediger einiges erhofft; e) Er beginnt, seine Predigten ähnlich anzulegen. An positiven Höreraktionen kann er merken, ob er auf dem richtigen Weg ist.

\section{Konkretion für die Predigt}

Den gleichen Prozeß, den dieser Prediger durchgemacht hat, sollten bei der Predigt auch die Hörer durchmachen - allerdings mit anderen Inhalten. Die Predigt sollte darum mit Fragen, Problemen, Interessen oder Neigungen der Hörer beginnen. Der Prediger kleidet sie in Worte. So weckt er das Interesse der Hörer (Motivation und Problemabgrenzung). Der gleichen Mühe muß sich auch der Journalist unterziehen, will er, daß seine Artikel gelesen werden. Die Ausführungen von Ewald Kley über das didaktische "Prinzip der Lücke" können die Funktion dieses Teiles noch mehr aufhellen: „Je mehr es einem Redner oder Autor gelingt, in seiner Einleitung eine alle seine Hörer und Leser erfassende, tragfähige Frage entstehen zu lassen, indem er vielleicht überzeugend die Lücke aufreißt, die er zu schließen sich vorgenommen hat, desto wirksamer ist sein Vortrag, seine Veröffentlichung, desto mehr ähnelt sein Reden oder Schreiben dem Unterricht. Weshalb es doch - entgegen weit verbreitetem Brauch - nützlich sein kann, Einleitung und Vorwort eines Buches nicht zu überblättern, sondern vielleicht sogar besonders gründlich zu lesen. ${ }^{\text {"15 }}$ Dann kann der Prediger Lösungsversuche aufzeigen, die der Hörer auf diese Fragestellung vielleicht schon unternommen hat oder noch unternehmen wird, die sich aber als falsch oder als Teillösungen herausstellen. In der nächsten Stufe ist dann das Angebot des Evangeliums oder des Glaubens aufzuzeigen, wie es konkret als Antwort auf die anfangs eingeführte Fragestellung Gestalt annimmt. In der letzten Stufe ist darzustellen, wie diese Antwort hilft, die genannten Fragen zu beantworten, die Probleme zu lösen, den Alltag zu gestalten.

Dieser Lernprozeß „Predigt“ spielt sich natürlich nicht in zehn Minuten am Sonntag ab. Vielmehr ist er eingebettet in ein Umfeld von Fragen, Suchen und Erfolg vor und nach der Predigt. Die Predigt ist der verbalisierte Lemprozeß, der in Worte kleidet, welche Fragen und Probleme der Predigt vorausgehen, der als Neuinformation ein Lösungsangebot macht und dann den möglichen Erfolg dieses Angebotes in Worten antizipiert. Zu diesem Aufbau ein Beispiel in Form eines gerafften Statements:

a) Die meisten Christen haben beim Stichwort "Nächstenliebe“ ein schlechtes Gewissen: zu oft rutscht ein böses Wort heraus, zu oft hat man Streit miteinander, zu oft stellen sich Konflikte ein, die man nicht ohne Härten harmonisieren kann, zu oft tut man Dinge aus Liebe, die sich im Nachhinein als falsch erweisen.

b) Die meisten wissen sich der Liebe verpflichtet, aber es gelingt ihnen nicht, entsprechend zu leben. Sie fühlen sich schuldig, wissen aber gleichzeitig nicht, wie sie das ändern sollen. 
c) Das folgende Beispiel kann verdeutlichen, warum man so of thilflos ist. Bei einem Gesprächsführungskurs wurde die Frage gestellt: Wie verhält man sich zu einem aggressiven Gesprächspartner. Eine ältere Dame antwortete: Christus hat gesagt, wir sollen ihn lieben. Hier liegt der Fehler. Mit dem Wort "Liebe“ auf den Lippen und einem Herz voll von gutem Willen allein kann man den Nächsten nicht lieben.

d) Liebe hat ihre konkreten Spielregeln. Eine von ihnen lautet: Überlegen, was der andere braucht.

e) Der andere braucht vielleicht einmal ein hartes Wort von mir. In der Erziehung muß ich oft das im Augenblick harte "Nein“ gebrauchen. Überlegen kann mir helfen zu merken: Der andere braucht nicht mein Portemonnaie, sondern meine Zeit. Wer überlegt, was der andere braucht, kann den Nächsten besser lieben; der braucht sich kein schlechtes Gewissen zu machen wegen Verhaltensweisen, die zwar hart sind, aber der Liebe entsprechen.

Die Anwendung der fünf Lernstufen kann auch das folgende Beispiel verdeutlichen. Es stammt aus dem Bereich der Werbung, die mit dem gleichen - in sich wertfreien - lernpsychologischen Instrumentarium arbeitet ${ }^{10}$ :

a) (weinendes Baby)

b) Manche Leute merken ganz genau, wenn mit ihrer Wäsche irgendwas nicht stimmt.

c) Man sollte immer ein Waschmittel nehmen, das die Wäsche nicht nur sauber wäscht oder nur gründlich oder nur weiß, sondern eins, daß die Wäsche rundherum perfekt wäscht.

d) Zum Beispiel $\mathrm{N}$ -

e) In $\mathrm{N}$ stecken die besten Waschmitteleigenschaften zu 100\% in der richtigen Kombination. Der Grund, warum wir sagen: Nur N ist $100 \% \mathrm{~N}$ (Lachen) - und warum immer mehr Leute auf $\mathrm{N}$ bestehen. (Babyweinen geht in Lachen über.)

Nimmt man die Predigt als Kommunikationsgeschehen ernst, ist Voraussetzung, daß alle Kommunikationspartner am Geschehen beteiligt sind. Predigt in der Kommunikationsform „Rede" beinhaltet immer die Gefahr, daß die nicht redenden Rezipienten aussteigen. Je mehr sie beteiligt sind, je mehr der Prediger Fragen der Hörer, ihre Stellungnahmen und Lösungsversuche aufgreift, desto mehr ist gewährleistet, daß die Predigt ein echter Kommunikationsprozeß ist. Dazu ist die Lernpsychologie in ihrer Anwendung eine geeignete Hilfe.

In diesem Zusammenhang wird eine Frage recht häufig gestellt: Muß denn eine Predigt immer diese fünf Punkte haben? Selbstverständlich kann es Variationen geben. Oft kann die Stufe „Versuch und Irrtum“ fehlen, oder sie findet sich als Gestaltungs- und Intensivierungsfaktor der Motivation. Auf eine Motivation sollte man allerdings nie verzichten, auch nicht, wenn man nach dem traditionellen Schrifterklärungs-Schema predigt; der Hörer muß wissen, warum die Ausführungen für ihn interessant und wichtig sind. Auf die Motivation kann man nur verzichten oder sie auf einen Satz reduzieren, wenn man von vornherein sicher ist: für dieses Thema sind alle motiviert.

Bei diesen Ausführungen darf ein in der Dichtung beheimatetes, in der Verkündigung übernommenes Stichwort nicht fehlen: Verfremdung. ${ }^{17}$ Verfremdung hat zum Ziel, vertraute Dinge fremd zu machen, damit ihr Informationswert wieder steigt. Verfremdung will redundante, nichtssagende Texte aus der Unverbindlichkeit des Un- 
auffälligen herausholen und zu Ursachen von Betroffenheit machen, will dadurch aufhorchen lassen und Aktivität anregen.18 Verkündigung, die Bibel reproduziert, läßt sie in der Unverbindlichkeit des Bekannten. Versucht sie aber, Bibel mit der Hörersituation in Verbindung zu bringen, sie in ihrer Aktualität für heute zu pointieren, verfremdet sie immer; denn wie die Situation des nächsten Jahres anders sein wird als die gegenwärtige, so wird auch die Botschaft im nächsten Jahr neu sein, wenn sie in diese Situation hinein verarbeitet wird. Das aber ist die Aufgabe der Verkündigung. Wie die Evangelisten in ihre jeweilige Gemeindesituation hinein die Botschaft von Christus verfremdet haben, so hat jede Zeit ihr fünftes Evangelium zu schreiben. ${ }^{10}$

\section{Konsequenzen für die Vorbereitung}

Solche Sicht der Predigt hat Auswirkungen auf die Vorbereitung von Predigten. Welcher Text steht für den Sonntag an? - Was will er sagen? - Welche Konsequenzen ergeben sich daraus? Diese Fragen reichen nicht aus. Die entsprechenden Leitfragen heutiger Verkündigung müssen lauten: Welche Probleme, Fragen Interessen, Neigungen usw. haben die Hörer, die am Sonntag in der Predigt sind? Entdecke ich im Text Verwandschaft zu diesen Fragen? Auf welche Fragen gibt der Text eine Antwort? Sind das Fragen und Themen von heute? Wie sieht die Antwort auf heutige Fragestellungen konkret aus, wenn man sie von der Bibel her geben will?20

Der Grad, in dem es dem Prediger bei der Vorbereitung gelingt, Hörersituation und Text der Schrift zueinander zu bringen, bestimmt den Grad gelungener Kommunikation und gelungenen Lernens bei der Predigt.

\section{Dialog und Kommunikationssituation bei der Predigt}

Wie bereits ausgeführt ist es notwendig, den Hörer in der Predigt zu Wort kommen zu lassen. Das zugeordnete Stichwort lautet „Dialog“. Er wird gefordert nicht nur durch das gesellschaftliche Umfeld der Demokratie, sondern auch durch ein hermeneutisches Vorverständnis, das Verkündigung nicht als Einweihung in die Geheimnisse eines Damals, sondern als Verlebendigung damaliger Botschaft in heutiger Situation versteht. Dialog ist notwendig. Aber verschiedene Kommunikationssituationen verlangen verschiedene Vollzugsformen. Das muß man sehen, soll Dialog nicht zum Statussymbol von "Möchte-gern-Demokraten" abmagern. Die entsprechende Form kann - so paradox das klingen mag - die monologischer Rede sein; denn Rede ist, so versteht es die Rhetorik seit langem, virtueller Dialog. ${ }^{21}$

Gruppengespräche als Vollzugsform der Predigt sind wohl nur in eucharistischen Kleingruppen möglich, die eine Zahl von ca. 6 bis höchstens 30 (z. B. Schulklassen) Teilnehmern nicht überschreiten dürfen. Halten solche Gruppen Gottesdienste, stehen sie meist nicht unter Zeitdruck. Das Gespräch kann sich in Ruhe entfalten. Im Rahmen eines normalen Sonntagsgottesdienstes stände einem Predigtgespräch nicht viel mehr als eine Viertelstunde zur Verfügung, und das bei erheblich größeren Teilnehmerzahlen.

Eine Kleingruppe ist, wählt sie die richtige Sitzordnung (Kreis, Oval - nicht Karree oder Längstisch), überschaubar: Jeder kann jeden sehen. Jeder kann beobachten, ob alle interessiert mittun. An Mimik und Gestik, durch Bestätigung oder Nachfrage 
kann die Teilnahme aller, auch der Stilleren, erkannt werden. In solchem Rahmen ist die Möglichkeit des Gesprächs gegeben, in dem das Thema im Miteinander zu einem gewissen Abschluß gebracht werden kann - möglicherweise nach dem methodischen Leitfaden der Lernstufen.

Dialogformen wie "Podiumspredigt" oder "Interviewpredigt“ erscheinen dem Ruf nach dialogischer Predigt nur in wenigen formalen Punkten gerecht zu werden. Oft stehen sie dialogischer Haltung und dialogischem Vollzug sogar sehr fern. Da mehrere Teilnehmer beteiligt sind, muß der Verlauf vorher abgesprochen sein. Dadurch blockiert man die Möglichkeit virtuellen Dialogs, wie sie in der monologischen Rede gegeben sind. Der Gemeinde wird Theater vorgespielt, Dialog wird vorgetäuscht, nicht realisiert ${ }^{22}$; oft erinnert der Fragende beim Interview, der doch Indentifikationsfigur für die Gemeinde sein soll, in peinlicher Weise an den mittelalterlichen „Stupido“. Es scheint allerdings so, als seien diese Formen inzwischen überholt. HansDieter Bastian hat recht behalten mit seiner Feststellung: „Hier verkauft ein geschickter Bauchredner seine Antworten über zwei Puppen. "23

Dagegen hat die monologische Rede durchaus ihre dialogische Stärke. Je größer die Zahl der Hörer ist, umso schwieriger ist es, sie an einem auch formal durchgeführten Dialog zu beteiligen. Der Prediger tut besser daran, den Dialog mit dem Hörer vorher zu führen, sei es, daß er sich in die Hörersituation hineindenkt und seine Eindrücke in Worte kleidet, sei es, daß er sich einen kleinen, repräsentativen Hörerkreis zu einem Vorbereitungsgespräch einlädt. ${ }^{24}$

Während der Predigt sind dialogische Kontakte über die sensorische Rückkoppelung oder das sog. pantomimische Feedback ${ }^{25}$ gegeben. Jeder Prediger und Redner kennt Ausdrucksformen, mit denen der Hörer sein Interesse oder Desinteresse, sein Verstehen oder Nichtverstehen, seine Zweifel, Zustimmung oder Ablehnung kundtut. Solche Ausdrucksmittel sind u. a.: Mimik, Kopfnicken, Gähnen, Einschlafen, Husten, Räuspern, Schneuzen, unruhiges Scharren mit den Füßen, Hin- und Herrutschen auf dem Platz, Tuscheln mit dem Nachbarn, Grinsen, Beifall, Zwischenrufe, protestartiger Abgang durch den Mittelgang. Der Prediger, der nicht ein Manuskript vorträgt, sondern frei spricht, weiß am Ende sehr gut, ob er mit seinen Hörern im Dialog gestanden hat, ob er sie angesprochen, an ihnen vorbei- oder über ihre Köpfe hinweggeredet hat.

Um das auch auf andere Weise zu erfahren, um gleichzeitig den frag- und gesprächswilligen Hörern die Möglichkeit zum Gespräch zu bieten, ist es zusätzlich sinnvoll, analog zum Vorgespräch die Möglichkeit eines Nachgesprächs anzubieten. Dieses hat die Funktion der lernpsychologischen Stufe fünf, der Verstärkung. Konkretionen werden im kleinen Kreis diskutiert.

Uber all das hinaus hat die monologische Rede noch eine spezifische Chance, die für die Predigt nicht zu unterschätzen ist. Will Predigt Daseinserhellung für die Menschen bringen, muß sie vom Menschen reden, auch vom Menschen mit seinen Schwächen, seinem Versagen, seinen Problemen. Bei diesem Ziel von Predigt müßten im Gespräch einzelne von sich reden - und wer mag das vor einer Gruppe, in der nicht das entsprechende Vertrauensklima herrscht? Eine Gesprächsrunde, in der sich die Einzelnen wenig kennen - und das wird in den Gemeinden häufig der Fall sein -, bietet zu wenig Schutz. 
Die Chance der Predigt liegt gerade darin, daß der Prediger solche persönlichen Erfahrungen seiner Hörer verbalisieren kann, daß der Hörer sich identifizieren kann („Da wird von mir gesprochen!"), ohne sich selbst ausliefern zu müssen. Das wird nur in sehr vertrauten Gruppen ein Gespräch leisten können. ${ }^{26}$

Im Rahmen kirchlicher Kommunikation ist darum für den Bereich "Predigt" die monologische Rede in ihrer Klassifikation als virtueller Dialog durchaus ein geeignetes Medium. Sein möglicher Mißbrauch ist kein grundsätzlicher Einwand.

\section{Anmerkungen:}

1. Siegfried Maser: Grundlagen der allgemeinen Kommunikationstheorie, Stuttgart 1972, S. $131 \mathrm{ff}$;; Vgl. auch: Elmar Maria Lorey: Mechanismen religiöser Information. MünchenMainz 1970, S. $64 \mathrm{ff}$.

2. Wilhelm Gössmann: Der Sprachgebrauch in der Kirche - kritisch, in: Communicatio socialis, Jahrg. 3 (1970), S. 1-12.

3. Diese Hermeneutik verdichtet sich etwa in dem folgenden Zitat aus Karl Barth: Homiletik, Zürich. 1966, S. 71: „Die Predigt ist nach Form und Inhalt unter allen Umständen gebunden als Schriftauslegung. Soll in der Kirche tatsächlich die Wahrheit geredet werden, so muß alles Reden in ihr in der Bewegung und Erwägung des Dokumentes dieser Wahrheit geschehen. Die Predigt darf nicht aus eigenem Reden quellen, sondern muß nach Form und Inhalt Schriftauslegung sein." - Dagegen Helmut Thieleke: Der Glaube des Evangeliums und die Wirklichkeit unserer Zeit, in: Universitas, Jahrg. 21 (1966), S. 1260: "Die Kirche droht einer dämonischen Selbstberuhigung zu verfallen, die mit dem Argument arbeitet: daß sie nicht verstanden werde, beruhe auf dem legitimen Ärgernis, das ein lauter verkündetes und darum befremdendes Evangelium ständig provozieren müsse. Sic sieht sich gerade durch das legitimiert, was sie anklagen müßte: daß sie nämlich ihre Botschaft esoterisch und selbstgenügsam zelebriert, statt sic zur Abholung und zu einem Auftrag selbstvergessenen Suchens werden zu lassen." - Vgl. auch Hans-Eckehard Bahr: Verkündigung als Information. Hamburg 1968, S. 105.

4. Vgl. Klaus Schwarzwäller: Leerlauf? Uber die Unfähigkeit von Kirche und Theologie, konkret zu werden. Neukirchen 1971.

5. Emil Dovifat: Handbuch der Publizistik, 3 Bde. Berlin 1968 ff., Bd. 1, S. 13 f.

6. Vgl. Karl Wilhelm Dahm: Beruf Pfarrer. München 1971, S. 315 ff.; Ders.: Kommunikationssoziologische Úberlegungen zur gegenwärtigen Predigtnot, in: E. Lange / P. Krusche / D. Rössler (Hrsg.), Predigtstunden IV, 2. Stuttgart-Berlin 1970, S. 9-20; Osmund Schreuder: Die Kirchengemeinde: Typen und Leitbilder, in: Otto Betz, Gemeinde von morgen. München 1969, S. 53-104; Ders.: Soziologische Aspekte der Verkündigung, Concilium, 4. Jahrgang (1968), S. $170 \mathrm{ff}$. Was Dahm und Schreuder in diesen Artikeln schreiben, hat die Diskussion um die Predigtöffentlichkeit vorangetrieben. Während jedoch beide Ansätze zu positiver Beurteilung offenlassen, pauschalisieren viele Oberflächliche diese Ergebnisse mit einer „und-überhaupt-Mentalität", um die Predigt als sinnlos auszuweisen.

7. Vor 1970 finden sich in homiletischen Büchern und Artikeln kaum Hinweise auf die Lernpsychologie.

8. Karl Wilhelm Dahm: Beruf Pfarrer, S. 317.

9. Vgl. Heribert Arens: Die Predigt als Lernprozeß. München 1972, S. 16 f.

10. Ebd. S. 73 ff., S. $94 \mathrm{ff}$.

11. Ebd. S. $95 \mathrm{ff}$. Die zweite Art der Lernprozesse bewegt sich im Rahmen der lernpsychologischen Stufe der Verstärkung (s. u.), die aber auch wiederum motiviert sein muß.

12. Vgl. dazu: Hans Löwe: Einführung in die Lernpsychologie des Erwachsenenalters. Berlin 1970, S. 39: „Die Aktivität des Subjekts darf nicht zu eng gefaßt werden. Lernen erfolgt nicht allein durch Tun, sondern auch durch das Nachdenken über das, was getan wurde bzw. getan werden soll. Aktivität bedeutet also nicht Unruhe, Beschäftigtsein oder gar Betriebsamkeit, sondern eine innere Aktivität, die das Lernen intensiviert."

13. Vgl. in der in Anm. 14 angegebenen Literatur die Abschnitte über "Motivation". 
14. Vgl. Werner Correll: Lernpsychologie. Donauwörth ${ }^{9} 1970$; Heinrich Roth: Pädagogische Psychologie des Lehrens und Lernens. Hannover 121970; Robert M. Gagné: Die Bedingungen menschlichen Lernens. Hannover 21970; in Ubertragung für die Verkündigung: Elmar Bartsch: Verkündigung als sakrale Leerformel, in: Harmut Bartsch: Entsakralisierung. München-Mainz 1970, S. 146 ff.; Heribert Arens: Die Predigt als Lernprozeß, a.a.O.

15. Ewald Kley: Das didaktische Prinzip der Lüdke zur Aktualisierung des kindlichen Interesses, in: H. Roth / A. Blumenthal (Hrsg.): Didaktische Analyse, Auswahl, Reihe A, Bd. 1. Hannover ${ }^{10} 1969,68-82$, S. 80 f.

16. Vgl. Heribert Arens, a.a.O., S. 142.

17. Vgl. Hans-Dieter Bastian: Verfremdung und Verkündigung. München 21967; Elmar Maria Lorey: a.a.O., S. 82 ff.; Heribert Arens: a.a.O., S. $138 \mathrm{ff}$.

18. Hans-Dieter Bastian: a.a.O., S. 46.

19. Ebd. vgl. besonders S. 8 ff., S. 47 f.

20. Vgl. Heribert Arens: a.a.O., S. 88 ff., S. $156 \mathrm{ff}$.

21. Vgl. Fritz Schweinsberg: Stimmliche Ausdrudksgestaltung im Dienste der Kirche. Heidelberg 1946, S. 329.

22. Vgl. Hans-Dieter Bastian: Theologie der Frage. München 1969, S. 320.

23. Ebd.

24. Zur Methodik solcher Gespräche ist lesenswert: Rolf Zerfaß: Der Anteil der Laien an der Predigt, in: Günter Biemer: Die Fremdsprache der Predigt. Düsseldorf 1970, S. $71 \mathrm{ff}$.

25. Vgl. Hellmut Geißner: Rede in der Offentlichkeit. Stuttgart 1969, S. 35.

26. Vgl. Heribert Arens: a.a.O., S. 118 ff.

\section{S U M M A R Y}

Because the sermon is addressed to a "captive ${ }^{\alpha}$ audience (an undemocratic method) and takes the form of a monologue, it is a poor means of communication. These three factors of the classic form of a means of address used in the Church show that the sermon is at the present moment undergoing a crisis. All the same, the authors consider the aims of the sermon: to give enlightenment con questions of living, and constantly to give pointers which are right and necessary. It is therefore worthwhile to work at improving the sermon technique, especially by methods drawn from educational psychology. The impetus given by these teaching methods can assure the force of the communication in a sermon, and increase its information content, and, in addition, can show how the sermon may be understood as part of the teaching process. If these methods are used regularly even the "monologue" form of preaching still has a chance, as eventually its purpose is to achieve „dialogue".

\section{RÉSUME}

Le sermon est pauvre en information, lu devant l'assemblée (et par là non-démocratique) et appartient au genre du monologue. Ces trois caractéristiques analytiques de la façon classique dont s'expriment les écclésiastiques montrent qu'à notre époque le sermon est en crise. Néanmoins, les auteurs tiennent le but du sermon: rendre plus clair le but de l'existence, pour constamment actuel, juste et nécessaire. Pour cela, il est bon de l'améliorer car, avec l'aide de l'enseignement de la psychologie, il est aussi capable d'amélioration. L'apprentissage des impulsion psychologiques peut assurer au sermon sa force de communication et augmenter son contenu informatique en même temps qu'il aide à saisir le sermon comme un procédé qui s'apprend. Alors, si l'on veut bien considérer l'importance de l'apprentissage de ces normes psychologiques, le sermon en tant qu'une forme du monologue a encore quelques chances car il tend au dialogue et l'atteint. 


\section{RES UMEN}

La predicación es pobre en contenido informativo, se realiza frente al público (es por lo tanto poco democrática) y omo género oratorio resulta un monólogo. Estas tres características analíticas de la clásica oratoria del locutor eclesástico muestran que la predicación es, en nuestra época, un fenómeno en crisis. No obstante los autores mantienen como válido el objectivo de la predicación: servir de constatación de su existencia - para poder permanecer actual, equilibrada y necesaria. Por ello es digna de ser mejorada y es mejorable, con ayuda de la psicología didáctica. Las sugerencias de la psicología didáctia con capaces de garantizar la capacidad de comunicación de la predicación y potenciar su contenido informativo, hasta que se aprenda a entender la predicación como proceso didáctio. Si se tienen en cuenta las leyes psicologico-didácticas, todavía le quedan probabilidades de sobrevivir a la predicación en forma de monólogo, pues entonces se proyecta y llega hasta el oyente con características de diálogo. 